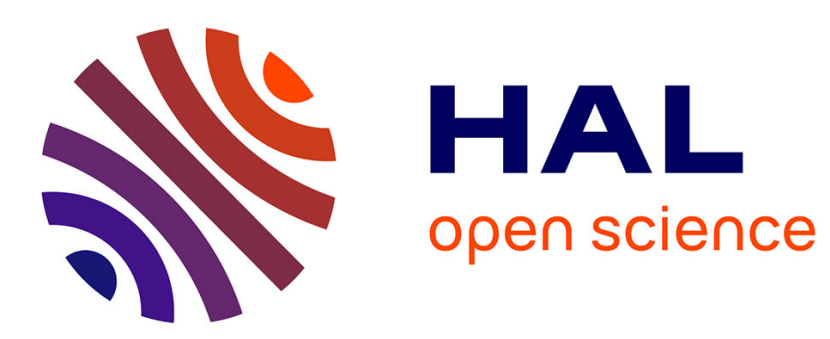

\title{
Enhancing and controlling parametric instabilities in mechanical systems
}

Alvaro Grandi, Suzie Protière, Arnaud Lazarus

\section{To cite this version:}

Alvaro Grandi, Suzie Protière, Arnaud Lazarus. Enhancing and controlling parametric instabilities in mechanical systems. Extreme Mechanics Letters, 2021, 43, pp.101195. 10.1016/j.eml.2021.101195. hal-03219590

\section{HAL Id: hal-03219590 \\ https://hal.science/hal-03219590}

Submitted on 6 May 2021

HAL is a multi-disciplinary open access archive for the deposit and dissemination of scientific research documents, whether they are published or not. The documents may come from teaching and research institutions in France or abroad, or from public or private research centers.
L'archive ouverte pluridisciplinaire HAL, est destinée au dépôt et à la diffusion de documents scientifiques de niveau recherche, publiés ou non, émanant des établissements d'enseignement et de recherche français ou étrangers, des laboratoires publics ou privés. 
Highlights

\section{Enhancing and controlling parametric instabilities in mechanical systems}

Alvaro A. Grandi,Suzie Protière,Arnaud Lazarus

- Modulating the energy landscape of a system in time induces parametric instabilities

- Operating close to an unstable equilibrium greatly enhance parametric instabilities

- Those extreme parametric instabilities allow us to design new synchronization rules

- We apply them to control the dynamics of an electromagnetic pendulum

- These concepts should allow for new dynamic functionalities in soft structures 


\title{
Enhancing and controlling parametric instabilities in mechanical systems
}

\author{
Alvaro A. Grandi ${ }^{a}$, Suzie Protière ${ }^{a}$ and Arnaud Lazarus ${ }^{a, *}$ \\ ${ }^{a}$ Institut Jean Le Rond d'Alembert, CNRS UMR7190, Sorbonne Université Paris, France
}

\section{ARTICLE INFO}

\section{Keywords:}

Dynamical systems

Instabilities

Parametric instability tongues

Control

Actuation

\begin{abstract}
A B S TRACT
We gain new fundamental insights on parametric instabilities that are at the heart of many physical phenomena from the dynamic buckling of slender structures in periodic compression to the emergence of Faraday waves or the spontaneous symmetry breaking in Floquet time crystals. Combining theoretical models and precision desktop experiments, we explain how to periodically vary the evolution function of a dynamical system to enhance and get control on parametric instabilities. We show on a proof of concept that is an electromagnetic pendulum: i) how to observe extremely high orders of parametric resonance, even in the presence of dissipation, ii) how to trigger and efficiently sustain the natural vibrations of an oscillator. The presented concepts being universal, they could offer new dynamical functionalities in various fields and at any scale, from actuation in soft robotics to vibrational motions in microelectromechanical resonators.
\end{abstract}

\section{Introduction}

Parametric instabilities can develop in any dynamical system whose local evolution function is periodically varied in synchrony with one of its natural time scale [1]. In practice, the evolution rule is varied by modulating a physical parameter of the system: a child can pump a playground swing by periodically squatting and standing, thus slightly varying the moment of inertia of the swing as a pendulum [2]; Faraday waves can emerge by vertically oscillating a layer of fluid, thus modulating the effective gravity felt by the perturbed surface waves $[3,4,5,6]$ and a slender structure under periodic compressive loads can dynamically buckle due to the small variations of its apparent flexural rigidity $[7,8]$. This concept being universal, parametric instabilities are encountered in various scientific areas from gravitational waves detectors [9] to plasma [10] or Micro Electro Mechanical Systems (MEMS) [11, 12]. By nature, parametric oscillators should allow for the design of complex stability diagrams in the modulation parameters space with numerous parametric instability tongues that could be exploited for promising dynamical functionalities. But apart in MEMS thanks to their extremely low damping [11, 13] and Floquet time crystals $[14,15]$, because they are many-body coupled oscillators with low dissipation [16], classical parametric instability regions are actually rather rare and barely controllable.

In this work, we investigate what features of parametric systems are essential to practically tailor the shape and number of their parametric instability tongues. We focus on a single linear oscillator and study the instability thresholds and dynamical responses of an electromagnetic pendulum to experimentally illustrate our theoretical concepts. We find that by carefully choosing the physical parameter to greatly

\footnotetext{
*Corresponding author

thumbnaibls.dnçs-email brineg @sorbonne-universite. fr (A.A. Grandi); suzie.protiere@sorbonne-universite.fr (S. Protière); arnaud. lazarus@sorbonne-universite.fr (A. Lazarus)

thuphasailsswwas-ur.tupipeg.q $r$ / alazarus/ (A. Lazarus)

$\operatorname{ORCID}(\mathrm{s})$ :
}

vary the evolution function of a linear dynamical system, it is possible to enhance and fully control parametric instabilities. For instance, it becomes simple to trigger extremely high super-harmonic instability regions or to practically use any periodic modulation functions to achieve interesting cyclic
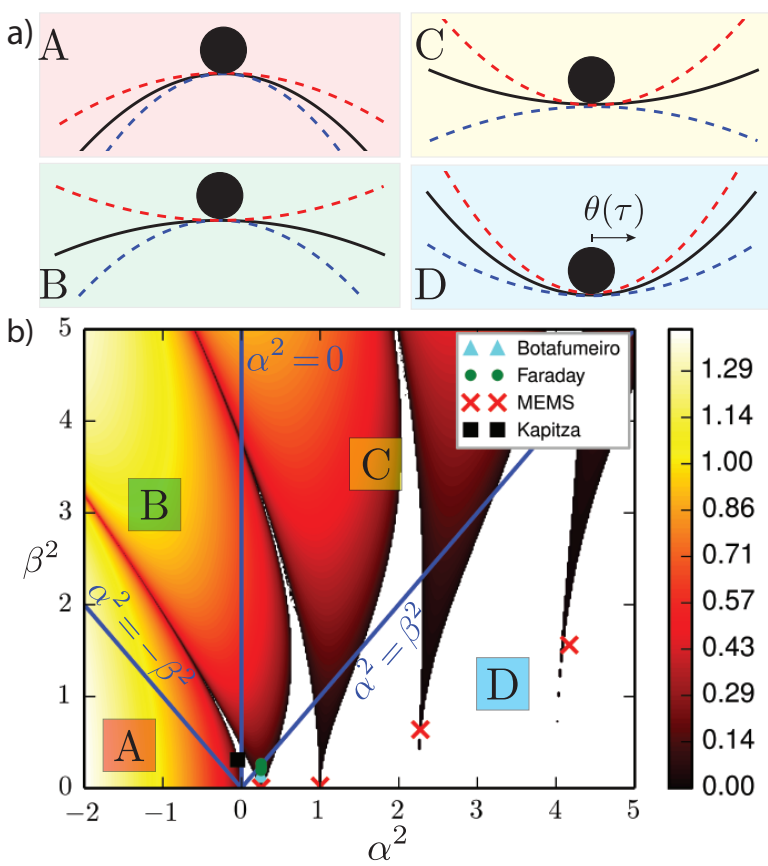

Figure 1: Linear stability of a mass locally moving in a harmonically varying potential $\mathcal{E}_{p}=\frac{1}{2}\left(\alpha^{2}+\beta^{2} \cos (\tau)\right) \theta^{2}$. a) Four qualitative scenarios depending on $\alpha^{2}$ and $\beta^{2}$. Black lines show $\mathcal{E}_{p}=\frac{1}{2} \alpha^{2} \theta^{2}$, red dashed lines $\mathcal{E}_{p}=\frac{1}{2}\left(\alpha^{2}+\beta^{2}\right) \theta^{2}$ and blue dashed lines $\mathcal{E}_{p}=\frac{1}{2}\left(\alpha^{2}-\beta^{2}\right) \theta^{2}$. A: $\alpha^{2}<0, \beta^{2}<-\alpha^{2}$. B: $\alpha^{2}<0$, $\beta^{2}>-\alpha^{2}$. C: $\alpha^{2}>0, \beta^{2}>\alpha^{2}$. D: $\alpha^{2}>0, \beta^{2}<\alpha^{2}$. b) Linear stability diagram of the Mathieu equation Eq.(1) in the modulation parameter space $\left(\alpha^{2}, \beta^{2}\right)$. Triangle, circle, cross, square represent classic experiments on parametric instability. 
motions. This work brings new physical insights on parametric instabilities and show that new dynamical functionalities can emerge when one periodically change the local evolution function about the equilibrium point of a dynamical system. The presented energetic concepts being universal, they could apply at any scale and in various fields, from electronics to physics or biology. In mechanics, possible applications range from the original realization of cyclic motions such as frequency dividers or clocks in MEMS to efficient dynamical actuation between equilibrium positions in soft robotics.

\section{From classical to extreme parametric systems}

The simplest linear model to get physical insights in the stability behavior of parametric systems is a mass locally moving in a harmonic potential whose curvature is periodically varying with time as illustrated in Fig.1a). Denoting $\theta(\tau)$ and $\dot{\theta}(\tau)$ the dimensionless position and velocity of the mass about the equilibrium $\theta(\tau)=\dot{\theta}(\tau)=0$, the total energy of the system can be expressed as the sum of a kinetic and potential part, respectively reading $\mathcal{E}_{c}=\frac{1}{2} \dot{\theta}^{2}$ and $\mathcal{E}_{p}=\frac{1}{2}\left(\alpha^{2}+\beta^{2} \psi(\tau)\right) \theta^{2}$ where $\psi(\tau)=\psi(\tau+\mathcal{T})$ is a $\mathcal{T}$-periodic modulation function between +1 and -1 and $\alpha^{2}$ and $\beta^{2}$ are the local curvatures of the potential. The linear equation of motion deriving from this energetic model reads

$$
\ddot{\theta}(\tau)+\left(\alpha^{2}+\beta^{2} \psi(\tau)\right) \theta(\tau)=0
$$

which is an Initial Value Problem in the form of a linear ODE with periodic coefficient. We recall the solution of Eq.(1) can be sought in the Floquet form $\theta(\tau)=p(\tau) e^{s \tau}+\bar{p}(\tau) e^{-s \tau}$ where $p(\tau)=p(\tau+\mathcal{T})$ is a $\mathcal{T}$-periodic complex eigenfunction and $s$ is a complex eigenvalue called the Floquet exponent $[17,18]$. Fig.1b) shows the numerical evolution of $\sigma=\max (\Re( \pm s))$ as a function of modulation parameters $\alpha^{2}$ and $\beta^{2}$ in the classic "Mathieu" case where $\psi(\tau)=\cos (\tau)$ and $\mathcal{T}=2 \pi$. The color dots $\sigma>0$ indicate solutions $\theta(\tau)$ diverging from $\theta(\tau)=0$, i.e. a particle that is locally unstable, when the white regions $\sigma=0$ indicate quasi-periodic oscillating solutions $\theta(\tau)$ about $\theta(\tau)=0$, i.e. a marginally stable particle.

The particular case $\beta^{2}=0$ corresponds to the classic harmonic oscillator where the mass is either marginally stable when $\alpha^{2}>0$ or locally unstable when $\alpha^{2}<0$. The generalized case $\beta^{2}>0$ models parametric oscillators and details on the rationalization of classic experiments by Eq.(1) are given in Appendix A. For $\alpha^{2}<0$, it is eventually possible to dynamically stabilize the naturally diverging mass $[18,19]$, but in a very small region of the modulation parameter space and only if $\beta^{2}>\left|\alpha^{2}\right|$, i.e. if the curvature of the potential is at least shortly positive (see cases A and B in Fig.1). The classic "Kapitza's pendulum" experiment $[20,21]$ that consists in stabilizing an inverted pendulum by modulating the gravitational energy potential through the vertical oscillation of its pivot point is indicated by a black square in the stability diagram of Fig.1b) [22]. The case $\alpha^{2}>0$ and $\alpha^{2}>\beta^{2}$ (case D in Fig.1) corresponds to classic parametric instabilities characterized by disconnected instability tongues whose tips originate, for $\beta \rightarrow 0$, at $\alpha=0.5 k$ with $k$ a positive integer. The $k^{t h}$ instability region indicates the emergence of $2 \mathcal{T}$ or $\mathcal{T}$-periodic motions depending if $k$ is odd or even, respectively. Classic experiments on the emergence of Faraday waves [23] or the parametric pumping of "O Botafumeiro" from Santiago de Compostella [2] are represented by green circles and blue triangles, respectively, in Fig.1b). Super-harmonic parametric instabilities in MEMS [13] are also represented in the same figure by red crosses. Finally, case $\mathrm{C}$ corresponds to periodic modulations that are so large, that the curvature of the potential in Fig.1a) is shortly negative. This is the case of few Faraday instability experiments when the acceleration of the shaker is such, than the effective gravity is negative for a short time, i.e. the fluid tends to vertically escape the recipient against gravity [23].

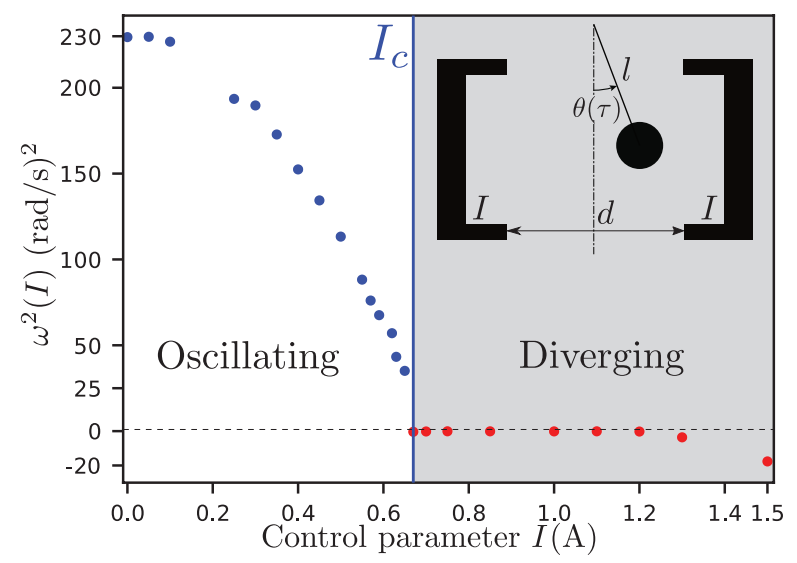

Figure 2: The experimental Floquet oscillator under study is a planar pendulum with a metallic marble that is symmetrically placed between two identical attracting electromagnets whose attracting force depends on the imposed electrical current $I$ (see sketch in inset). The scalar $\omega^{2}(I)$, characterizing the local evolution function of the electromagnetic pendulum, drastically depends on the constant control parameter $I$.

It is clear from Fig.1b) that parametric instabilities usually exploited in mechanical Floquet systems are the ones close to the tips of the instability tongues. In fact, when periodically varying the length of a pendulum, the acceleration of a shaker or the membrane stiffening of a micrometric plate, it is either impractical or energetically too costly to maintain a large variation of the evolution function of a system over relatively long periods of time, i.e. having both large $\beta^{2}$ and $\alpha^{2}$. And since because of inherent damping, there is an exponential narrowing of the tips of the tongues with increasing instability region number $k$ [11], macroscopic systems are typically limited around the first instability region and higher orders parametric resonances have only been observed in MEMS thanks to their extremely large quality factors $[11,13]$. In Fig.2, we propose an experiment where 
parametric instabilities are enhanced. The setup consists of a $2 D$ pendulum: a metallic marble with radius $0.6 \mathrm{~cm}$ is attached to a string of length $l=4.3 \mathrm{~cm}$. The marble is symmetrically placed between two electromagnets (with typical holding force of $1000 \mathrm{~N}$ ) that are separated by a distance $d=4 \mathrm{~cm}$. Upon a given electrical current $I$, the magnets are turned $\mathrm{ON}$ and both attract the marble. The local evolution function of this electromagnetic pendulum is characterized, for a given control parameter $I$, by locally perturbing the pendulum from its trivial vertical equilibrium position with a small initial angle $\theta(0)$ at time $t=0$. We find linear angular responses can be written in the form $\theta(t)=$ $\theta(0) \Re\left(e^{-i \sqrt{\omega^{2}(I)} t}\right)$ where $\omega^{2}(I)$ is shown in Fig.2. Two qualitative responses are observed: below $I<I_{c}$, the pendulum is harmonically oscillating in the form $\theta(t)=\theta(0) \cos (\omega(I) t)$ with a natural frequency $\omega(I)$ that decreases as $I$ increases. Above a critical current $I_{c}$, the pendulum locally diverges in the form $\theta(0) e^{\omega(I) t}$, eventually choosing one or the other side depending of initial symmetry imperfections. Because the bifurcation at $I=I_{c}$ is sub-critical, there is always a "jump" of $\omega^{2}(I)$ around $I_{c}$ where our experiment is imperfection sensitive.

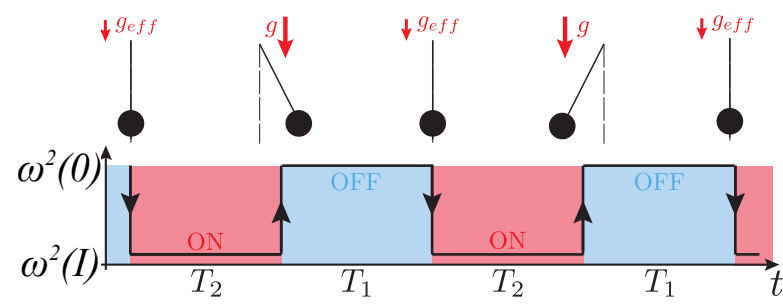

Figure 3: Sketch of the input-output synchronization that would be required for parametric pumping in the case of a time-periodic system with a square wave modulation function $T_{1}=T_{2}=T / 2$. To amplify any small perturbation about the equilibrium $\theta(t)=0$, one needs to turn the magnets ON (decrease effective gravity) when the pendulum height is minimal, just before the mass goes up, and turn them OFF (put back strong gravity) when the pendulum height is maximum, just before the mass goes down.

\section{Beyond the tip of the parametric instability tongues}

Unlike classical parametric systems, it is straightforward with the system of Fig.2 to fully explore regions $A-D$ of Fig. 1b) by periodically varying the electrical current $I$ below or above $I_{c}$. In this work, we modulate $\omega^{2}(I(t))$ in a square wave fashion as shown in Fig.3 (case D) and 5a) (case C). Precisely, we turn the electromagnets $\mathrm{OFF}(I=0)$ and ON ( $I \neq 0$ ) during $T_{1}$ and $T_{2}$ seconds, respectively, so that the modulation period is $T=2 \pi / \Omega=T_{1}+T_{2}$. The linear equation of motion of this two-state oscillator reads simply

$$
\begin{cases}\ddot{\theta}(t)+\omega^{2}(0) \theta(t)=0 & \text { during } T_{1}, \\ \ddot{\theta}(t)+\omega^{2}(I) \theta(t)=0 & \text { during } T_{2} .\end{cases}
$$

Introducing the dimensionless time $\tau=\Omega t$, the square wave modulation function $\psi(\tau)=+1$ during $\mathcal{T}_{1}=2 \pi T_{1} / T$ and $\psi(\tau)=-1$ during $\mathcal{T}_{2}=2 \pi T_{2} / T$ and the dimensionless modulation parameters

$$
\alpha^{2}=\frac{\omega^{2}(0)+\omega^{2}(I)}{2 \Omega^{2}}, \quad \beta^{2}=\frac{\omega^{2}(0)-\omega^{2}(I)}{2 \Omega^{2}},
$$

Eq.(2) can be reduced in the dimensionless form of Eq.(1) which, in the case of a piecewise modulation function $\psi(\tau)$, is called the Meissner equation [1]. On top of being easy to experimentally implement, a square wave parametric oscillator can be analytically solved [24, 25]. Notably, introducing $\lambda=i \sqrt{\alpha^{2}+\beta^{2}}$ and $\eta=i \sqrt{\alpha^{2}-\beta^{2}}$, the analytical growth rate $\sigma=\max (\Re( \pm s))$ of the Floquet forms of Eq.(1) reads $\sigma=\left|\Re\left(\frac{1}{2 \pi} \ln \left(\frac{\Delta \pm \sqrt{\Delta^{2}-4}}{2}\right)\right)\right|$ where

$$
\Delta=2 \cosh \left(\lambda \mathcal{T}_{1}\right) \cosh \left(\eta \mathcal{T}_{2}\right)+\left(\frac{\eta}{\lambda}+\frac{\lambda}{\eta}\right) \sinh \left(\lambda \mathcal{T}_{1}\right) \sinh \left(\eta \mathcal{T}_{2}\right)
$$

Evolution of $\sigma$ is shown in Fig.4a) for $T_{1}=T_{2}=T / 2$ in an extremely extended parameter space $\left(\alpha^{2}, \beta^{2}\right)$ (two order of magnitude more as compared to the stability chart of Fig.1b)). We recognize the $k^{\text {th }}$ instability tongues originating at $\alpha=0.5 k$ for $\beta \rightarrow 0$ like for the Mathieu equation, although the tongues are here tailored in discrete pockets. We found that the discrete location of those instability pockets can be rationalized by the input-output synchronization sketched in Fig.3. Parametric pumping is achieved by dropping the effective gravity (magnets $\mathrm{ON}$ ) when the pendulum has minimal potential energy, i.e. at $\theta(t)=0$, and putting back strong gravity (magnets OFF) when potential energy is maximum, i.e. when $\theta(t)$ is an extremum. For example, Fig. 3 illustrates the most efficient pumping at the origin of the classic primary instability tongue, that consists in turning the magnets ON and OFF every quarter of a cycle of the pendulum's response. But many higher super-harmonic modes of parametric amplification are theoretically possible that can verify the aforementioned input-output synchronization. Knowing the electromagnetic pendulum has a natural period $2 \pi / \sqrt{\alpha^{2}+\beta^{2}}$ when OFF during $\mathcal{T}_{1}=\pi$ and $2 \pi / \sqrt{\alpha^{2}-\beta^{2}}$ when ON during $\mathcal{T}_{2}=\pi$, those modes need to fulfill

$$
\frac{(2 m-1) 2 \pi}{4 \sqrt{\alpha^{2}+\beta^{2}}}=\pi \quad \text { and } \quad \frac{(2 n-1) 2 \pi}{4 \sqrt{\alpha^{2}-\beta^{2}}}=\pi
$$

where $m$ and $n$ are positive integers and $m>n$, i.e. $\alpha^{2}>$ $\beta^{2}$. As the mode numbers $(m, n)$ are varied, Eq.(5) gives us a new discrete design rule in the $\left(\alpha^{2}, \beta^{2}\right)$ space to easily locate the center of the instability pockets as shown by the grey dots in Fig.4a). Physically, $(2 m-1)$ and $(2 n-1)$ represent the number of quarters of a cycle of the pendulum's response one can fit in the modulation periods $T_{1}$ and $T_{2}$, respectively. Fig.4b) shows four experimental dynamical responses whose transient regimes are representative of the many parametric instabilities observed in the electromagnetic pendulum of Fig.2. As expected, the parametric 

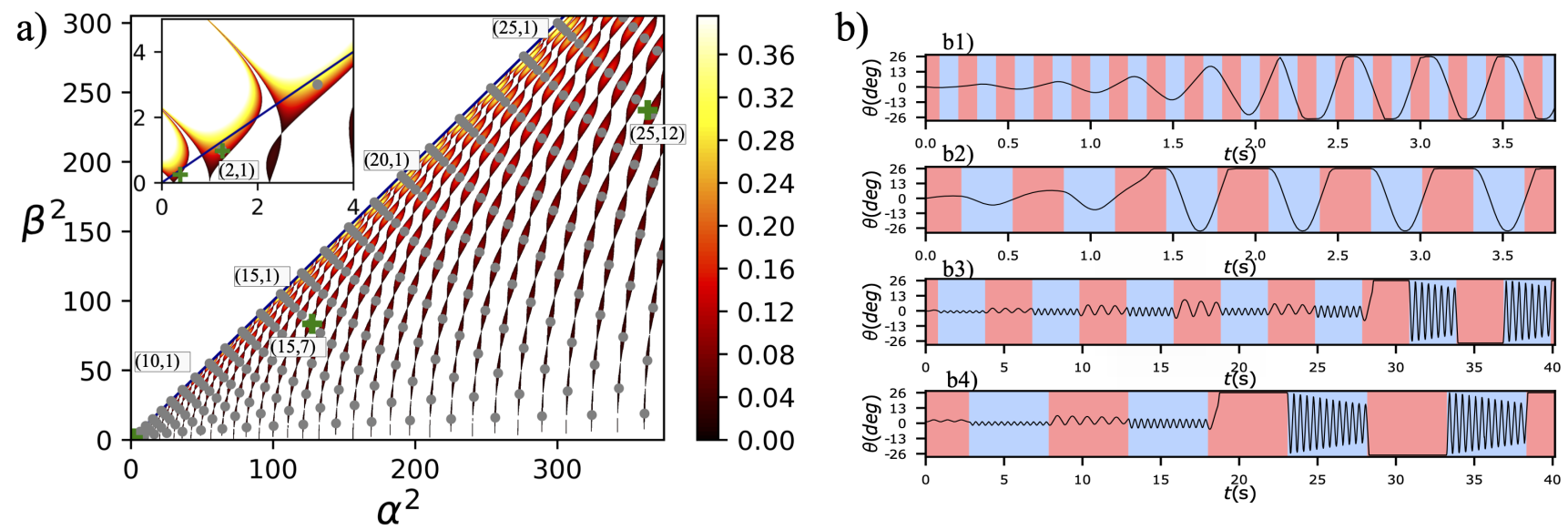

Figure 4: Triggering extremely high orders of parametric resonances. a) Stability diagram of the Meissner equation Eq.(1) showing $\sigma$, up to 0.4 , in the extremely extended $\left(\alpha^{2}, \beta^{2}\right)$ space. Grey dots $(m, n)$ represent the discrete solutions of Eq.(5). Green crosses represent the experimental parameters expressed in the $\left(\alpha^{2}, \beta^{2}\right)$ space. Inset zooms on the classic first instability regions. b) Dynamical responses of the electromagnetic pendulum of Fig. 2 when $\omega^{2}(I(t))$ is modulated in a square wave fashion with $T_{1}=T_{2}=T / 2$. b1) A classic " $(1,1)$ " parametric amplification obtained for $\omega(0)=\sqrt{g / l} \approx 15.15 \mathrm{rad} / \mathrm{s}, \omega(I) \approx 6.8 \mathrm{rad} / \mathrm{s}$ and $T \approx 0.33 \mathrm{~s}$. b2) A $(2,1)$ parametric instability obtained for $\omega(0) \approx 15.15 \mathrm{rad} / \mathrm{s}, \omega(I) \approx 6.8 \mathrm{rad} / \mathrm{s}$ and $T \approx 0.62 \mathrm{~s}$. b3) A (15,7) parametric pumping obtained for $\omega(0) \approx 15.15 \mathrm{rad} / \mathrm{s}, \omega(I) \approx 6.5 \mathrm{rad} / \mathrm{s}$ and $T \approx 6.02 \mathrm{~s}$. b4) A (25, 12) parametric amplification obtained for $\omega(0) \approx 15.15 \mathrm{rad} / \mathrm{s}, \omega(I) \approx 6.5 \mathrm{rad} / \mathrm{s}$ and $T \approx 10.16 \mathrm{~s}$.

pumpings at the origin of the observed limit cycles are in good qualitative agreement with the synchronization principle illustrated in Fig.3. Expressing the experimental modulation parameters $\omega(I)$ and $\Omega$ associated with the responses of Fig.4b) in the dimensionless form $\alpha^{2}$ and $\beta^{2}$ thanks to Eq.(3), we report experimental data (green crosses) in the stability diagram of Fig.4a) and find excellent agreement with the expected $(m, n)$ parametric pumping mode from Eq.(5). The parametric instability of Fig.4b1), found in the classic primary instability region $k=1$, leads to a sub-harmonic $2 T$-periodic limit cycle. Fig.4b2) corresponds to a $(m, n)=$ $(2,1)$ unstable parametric mode, located in the instability region $k=2$, and leads to a $T$-periodic limit cycle as expected since $m$ is even (see movie 1 in [26]). Fig.4b3) and Fig.4b4) display a $(15,7)$ and $(25,12)$ parametric pumping mode respectively, leading to extremely high super-harmonic $2 T$ periodic motions since $m$ is odd in both cases. Since the instability region number follows $k=m+n-1$, the response of Fig.4b4) (see movie 2 in [26]) is actually located in the $36^{\text {th }}$ instability tongue as shown in Fig.4a); an achievement since, to our knowledge, the record $k=28$ was observed in a MEMS in 2016 [13]. It is beyond the scope of this paper to report the plethora of parametric instabilities existing in this fundamental electromagnetic pendulum, as suggested by the impressive number of instability pockets in Fig.4a), but the various motions displayed in Fig.4b) already highlights the potential of extreme parametric instabilities for new dynamical control in mechanical systems.

\section{Triggering and sustaining a natural oscillation}

Another overlooked limit that could be useful in mechanical Floquet systems is the almost "impulse train" case illustrated in the sketch of Fig.5a). Here, the magnets are almost continuously OFF, except for every period $T_{1} \approx T$ where they are turned shortly ON during $T_{2}$ with a current $I$. Our system is a locally stable pendulum, characterized by a harmonically damped oscillating perturbation with natural pe$\operatorname{riod} T_{0}=2 \pi / \omega(0)$, except every period $T_{1}$ where the local evolution function of the pendulum is shortly but drastically changed. This original scenario is still theoretically described by the governing equations Eqs.(1)-(4). Notably, the analytical growth rate $\sigma$ as a function of $\left(\alpha^{2}, \beta^{2}\right)$ is given in Fig.5a) for $T_{1}=0.98 T$ and $T_{2}=0.02 T$. Now the parametric instability tongues are thin parallel regions whose "left" frontiers correspond to the black lines $\sqrt{\alpha^{2}+\beta^{2}}=0.5 k$, that have been obtained by introducing the limits $\mathcal{T}_{2} \rightarrow 0$ and $\mathcal{T}_{1} \rightarrow 2 \pi$ in Eq.(4). This stability diagram actually means one should be able to parametrically amplify the harmonic perturbation with natural period $T_{0}$ as soon as $T \approx 0.5 k T_{0}$, where $k$ is the positive integer representing the instability tongue number.

As a proof of concept, we show in Fig.5b) the experimental response of the electromagnetic pendulum (characterized by a natural period $T_{0}=2 \pi / \sqrt{g / l}=412 \mathrm{~ms}$ and a natural frequency $f_{0}=1 / T_{0}=2.43 \mathrm{~Hz}$ ) when the magnets are continuously OFF except every $T \approx T_{1} \approx 1220 \mathrm{~ms}$ where $I \approx 1.2$ A during $T_{2} \approx 25 \mathrm{~ms}$ (see movie 3 in [26]). Expressing the experimental parameters $\omega(0)=2 \pi f_{0}, \omega(I)$ and $\Omega=2 \pi / T$ in the dimensionless form $\alpha^{2}$ and $\beta^{2}$ thanks to Eq.(3), we report experimental data (green cross) in the 


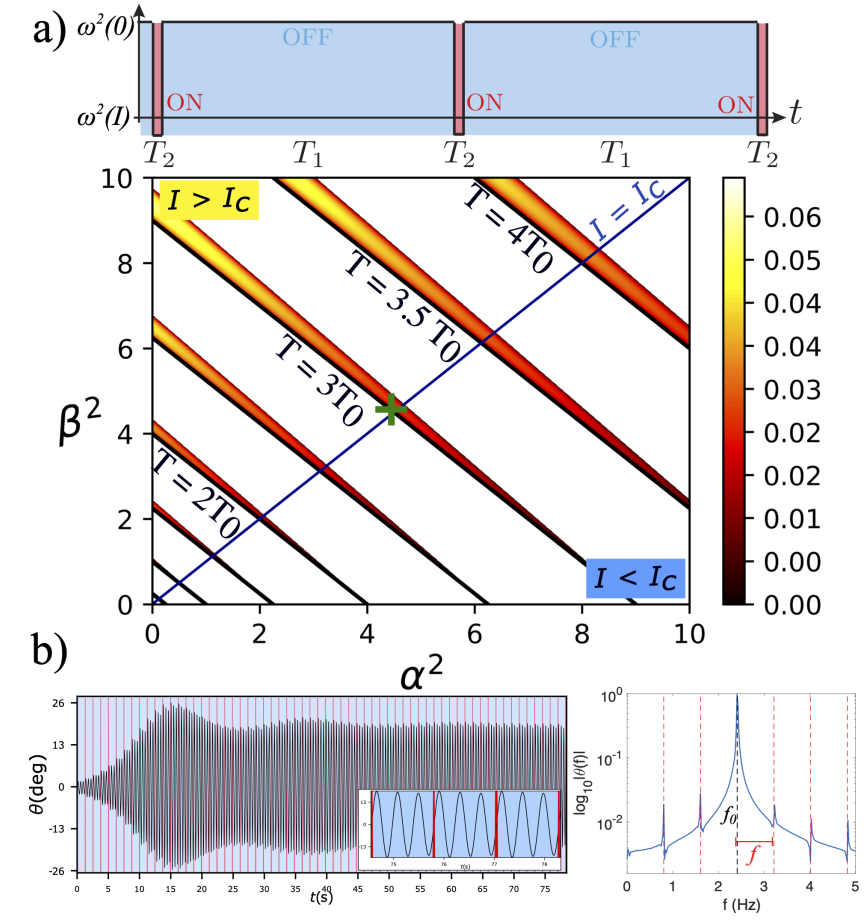

Figure 5: Triggering a "natural" limit cycle. a) Stability diagram of the Meissner equation Eq.(1) in the "impulse train" case when $T_{1}=0.98 T$ and $T_{2}=0.02 T$. Black lines show the $\operatorname{limit} T_{1} \rightarrow T$ and $T_{2} \rightarrow 0$. The green cross represents the experimental modulation parameters associated with the response shown in b) Left: experimental response observed when the electromagnetic pendulum is OFF during $T_{1} \approx 1.22 \mathrm{~s}$ and $\mathrm{ON}$, with $I \approx 1.2 \mathrm{~A}\left(I>I_{c}\right)$ during $T_{2} \approx 0.025 \mathrm{~s}\left(\approx 0.02 T_{1}\right)$. Inset zooms on the $T$-periodic limit cycle. Red lines represent the very short time the magnets are ON. Right: Fast Fourier Transform of the permanent regime of the response. The black dashed-line represents the value of the natural frequency of the pendulum when the red ones represent the secondary harmonics $f_{0} \pm h f$, where $f=1 / T$ and $h$ is an integer.

stability diagram of Fig.5a). We find, as expected since the applied modulation period $T$ is $6^{\text {th }}$ time slower than half the natural period of the pendulum, that the limit cycle of Fig.5b) results from a parametric resonance of order $k=6$. Since $k$ is even, we expect to trigger a $T$-periodic limit cycle which is confirmed by the FFT of the experimental permanent regime in Fig.5b) that shows spectral rays located every $f_{0} \pm h f$ with $h$ an integer and $f=1 / T$. Interestingly, the limit cycle is very close to a purely sinusoidal motion with a fundamental frequency $f_{0}$ since the amplitudes of the secondary harmonics weight no more than $3 \%$ of the fundamental one. Actually, as illustrated by the inset of Fig.5b) that displays the last three periods of the recorded experimental response, the triggered limit cycle is the free damped oscillation of the pendulum with natural period $T_{0}$ that is "reset" every $3 T_{0}$ thanks to the synchronized impulse of input energy represented by red vertical lines. Every $T \approx 0.5 k T_{0}$, the same periodically reseted natural sinusoidal motion could be triggered and sustained with the difference that the growth rate of the transient oscillations decreases with $k$ until eventually no instability occurs for large $k=k_{\max }$ because of insufficient energy exchanges (see Appendix B for the case $T \approx 6 T_{0}$ ). To increase the maximum order of observed parametric resonance $k_{\max }$, one could increase the $Q$ factor of the pendulum or the current intensity $I$. Interestingly, unlike the classic resonance phenomenon, the bandwidth of this parametric resonance, i.e. the width of the instability tongues of Fig.5a), depends on the "width" of the imposed impulse train and not on the $Q$ factor: the shorter the impulse train, the shorter the periodic energy exchange and the thinner the instability regions. Another useful property is the tuning capabilities offered by this system: by maintaining the magnets almost continuously ON with $I<I_{c}$ instead of OFF with $I=0$ as in Fig.5a), one could efficiently trigger and sustain any sinusoidal oscillations with angular frequency $\omega(I)$ between $\omega(0)$ and almost 0 , as suggested by Fig.2. For systems like MEMS that are tiny beam or plate resonators with enormous $Q$ factors (because they operate in Ultra High Vacuum) driven by periodic electrostatic fields, an application of this particular parametric pumping could allow to efficiently generate highly super-harmonic sinusoidal signals with extremely tunable fundamental frequencies.

\section{Conclusions and perspectives}

Based on a better fundamental understanding of parametric oscillators, we have shown through theoretical and experimental proofs of concepts, how to extremely enhance and better control parametric instabilities. The presented concepts being universal, they could apply to any dynamical systems with a natural cycle which time scale could be periodically varied: from resonant MEMS to purely electronic circuits or lasers where researchers are often seeking for new dynamical functionalities to exploit, but also non engineered systems such as business cycles or circadian rhythms.

Quasi-static symmetry breaking like buckling allows, through the slow variation of a single parameter, to switch from one unstable to a new stable equilibrium configuration, albeit with a unique time scale mainly determined by the growth rate of the diverging instability. Parametric instability is a dynamic symmetry breaking that, upon the periodic variation of a parameter, should allow for even more control on switchings between equilibrium or time periodic configurations, as soon as one modulates the evolution function of a system close to its diverging instability. Since elastic buckling has been shown to enable actuations of soft machines [27] or structures [28], the application of extreme parametric instabilities to those fields should improved actuation, especially in time.

\section{Declaration of competing interest}

The authors declare that they have no known competing financial interests or personal relationships that could have appeared to influence the work reported in this paper. 


\section{Acknowledgments}

A. Lazarus and S. Protière are grateful for financial support from Sorbonne University (EMERGENCES grants).

\section{A. Rationalization of the instability thresholds of classical parametric systems}

We rationalize various experiments from the literature with the linear parametric oscillator governed by Eq.(1) of the manuscript. All experimental data can then be represented in the same stability diagram.

\section{A.1. Faraday instability}

The first experimental demonstration of a parametric behavior was made by Faraday in 1831. His experiment consists in creating a wave motion in a fluid by vertically oscillating it.

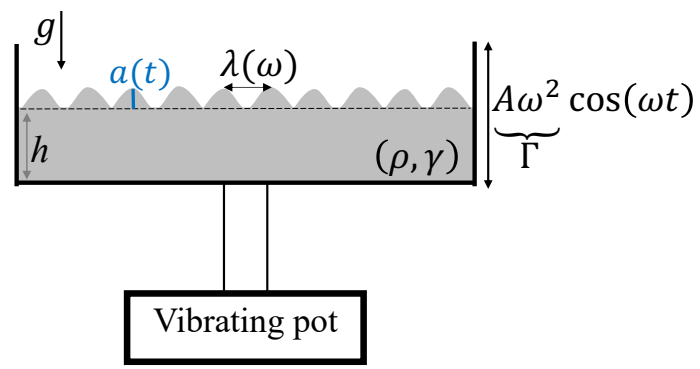

Figure 6: Experimental setup to observe Faraday instability. A fluid is placed over a vibrating pot with a frequency of excitation $\omega$ and an acceleration of the excitation $\Gamma$. The fluid is characterized by : $a(t)$ the amplitude of displacement of the surface, $h$ the depth of the fluid, $\gamma$ the surface tension, $\rho$ the density of the fluid, $\lambda$ the wavelength and $g$ the gravitational acceleration.

Based on the work of Benjamin and Ursell [4] and considering only the first mode, the evolution of the amplitude of displacement of the surface of the fluid for the Faraday instability reads

$$
\frac{d^{2} a(t)}{d t^{2}}+k \tanh (k h)\left(\frac{k^{2} \gamma}{\rho}+g-\Gamma \cos (\omega t)\right) a(t)=0
$$

where $k=2 \pi / \lambda$ is the fluid wavenumber and the dispersion equation for capillaro-gravitational waves is

$$
\omega=\sqrt{k \tanh (k h)\left(g+\frac{k^{2} \gamma}{\rho}\right)} .
$$

By implementing a change of variable $\tau=\omega t$ and using a first approximation for small angles $\theta(\tau)=a(\tau) / \lambda$, we are able to write Eq.(1) in the standard dimensionless form of the manuscript:

$$
\ddot{\theta}(\tau)+\left(\alpha^{2}-\beta^{2} \cos (\tau)\right) \theta(\tau)=0
$$

with $\alpha^{2}=\frac{k \tanh (k h)}{\omega^{2}}\left(g+\frac{k^{2} \gamma}{\rho}\right)$ and $\beta^{2}=\frac{k \tanh (k h) \Gamma}{\omega^{2}}$.

\begin{tabular}{|l|l|l|}
\hline Experimental parametric parameters & $\alpha^{2}$ & $\beta^{2}$ \\
\hline$f=20 \mathrm{~Hz} ; \Gamma=g \mathrm{~m} . \mathrm{s}^{-2}$ & 0.249 & 0.196 \\
$f=80 \mathrm{~Hz} ; \Gamma=4 \mathrm{~g} \mathrm{~m} . \mathrm{s}^{-2}$ & 0.250 & 0.201 \\
$f=120 \mathrm{~Hz} ; \Gamma=7.5 \mathrm{~g} \mathrm{~m} . \mathrm{s}^{-2}$ & 0.251 & 0.232 \\
$f=160 \mathrm{~Hz} ; \Gamma=12.5 \mathrm{~g} \mathrm{~m} . \mathrm{s}^{-2}$ & 0.250 & 0.267 \\
\hline
\end{tabular}

Table 1

Experimental parameters for the Faraday instability used by Protière [23] and corresponding values of the dimensionless modulation parameters $\alpha^{2}$ and $\beta^{2}$. Data points presented in Fig.1 of the manuscript.

An experimental study of Faraday instability is presented in the work of Protière [23]. The properties of the fluid are: surface tension $\gamma=0.0209 \mathrm{~N} / \mathrm{m}$, density $\rho=0.965 \times 10^{3}$ $\mathrm{kg} . \mathrm{m}^{-3}$, the depth of the fluid is $h=4 \times 10^{-3} \mathrm{~m}$ and the gravitational acceleration is $g=9.81 \mathrm{~m} . \mathrm{s}^{-2}$. Using the dispersion equation of capillary-gravitational waves Eq.(7) with the explicit form of parametric parameters of Eq.(8), we are able to calculate in Table 1 the dimensionless parameters $\left(\alpha^{2}, \beta^{2}\right)$ for each experimental point that are then reported in the Fig.1 of the manuscript.

\section{A.2. Parametric pendulum}

The system is a pendulum where the pivot point is vertically oscillating as shown in Fig.2.

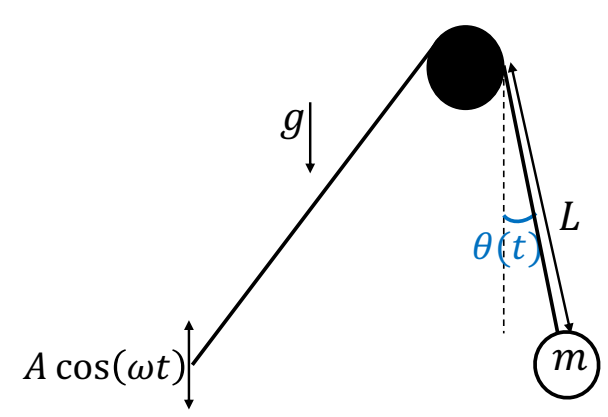

Figure 7: Experimental setup and parameters for a parametric pendulum. The frequency of excitation is $\omega$ and the amplitude of excitation is $A$. The pendulum is characterized by its length $L$, mass $m$ and $\theta(t)$ the angular displacement measured from the vertical position. The gravitational acceleration is $g$.

The undamped equation of motion for this system is the following

$$
I \frac{d^{2} \theta(t)}{d t^{2}}+m L\left(g-A \omega^{2} \cos \omega t\right) \sin \theta(t)=0
$$

where $I$ is the moment of inertia and the rest of the parameters are defined on Fig.2. In this case parametric instabilities are used to trigger the motion of the pendulum in the equilibrium position $\theta=0$. By linearizing Eq.(9) about $\theta=0$ (approximation of small angles for the angular displacement $\theta(t))$ and upon the change of variable $\tau=\omega t$, we are able 


\begin{tabular}{|l|l|l|}
\hline Experimental parametric parameters & $\alpha^{2}$ & $\beta^{2}$ \\
\hline$f=0.22 \mathrm{~Hz} ; A=2.9 \mathrm{~m}$ & 0.244 & 0.141 \\
\hline
\end{tabular}

Table 2

Experimental parameters used by San Martin [2] and corresponding values of the dimensionless modulation parameters $\alpha^{2}$ and $\beta^{2}$. Data point presented in Fig.1 of the manuscript.

to write Eq.(9) in the form of the Mathieu equation of the manuscript:

$$
\begin{aligned}
& \ddot{\theta}(\tau)+\left(\alpha^{2}-\beta^{2} \cos (\tau)\right) \theta(\tau)=0 \\
& \text { with } \alpha^{2}=\frac{g}{L} \frac{1}{\omega^{2}} \text { and } \beta^{2}=A \frac{1}{L} .
\end{aligned}
$$

An experimental example of this system is the giant censer ("O Botafumeiro") from Santiago de Compostella presented in the work of San Martin [2]. The length of the pendulum is $L=20.6 \mathrm{~m}$, the mass is $m=56.5 \mathrm{~kg}$ and the gravitational acceleration is $g=9.81 \mathrm{~m} . \mathrm{s}^{-2}$. The dimensionless experimental parameters are calculated using the experimental values of San Martin [2] and Eq.(10) and are reported in Table 2.

\section{A.3. Inverted pendulum}

The system is an inverted pendulum where the pivot point is vertically oscillating (the displacement is often imposed by a shaker). In this case parametric instabilities are used to stabilize the naturally diverging equilibrium.

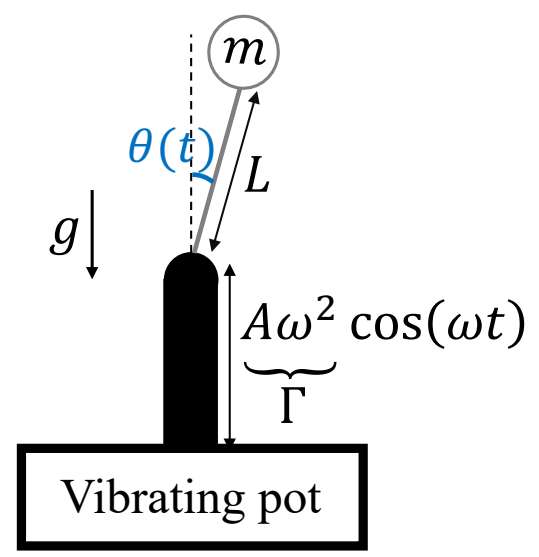

Figure 8: Experimental setup and parameters for stabilizing an inverted pendulum. The inverted pendulum is placed over a shaker with a frequency of excitation $\omega$, an amplitude of excitation $A$ and an acceleration of excitation $\Gamma=A \omega^{2}$. The pendulum is characterized by its length $L$, mass $m$ and $\theta(t)$ is the angular displacement measured from the vertical position. The gravitational acceleration is $g$.

The undamped equation of motion for this system is the same presented for the parametric pendulum Eq.(9) but in this case the considered equilibrium position is $\theta=\pi$. By linearizing Eq.(9) about this equilibrium position (approximation of small angles for the angular displacement $\theta(t)$ ) and upon the change of variable $\tau=\omega t$, we are able to write

\begin{tabular}{|l|l|l|}
\hline Experimental Parametric parameters & $\alpha^{2}$ & $\beta^{2}$ \\
\hline$f=157.17 \mathrm{~Hz} ; \Gamma=61.3 \mathrm{~m} . \mathrm{s}^{-2}$ & 0.050 & 0.31 \\
\hline
\end{tabular}

Table 3

Experimental parameters used by Smith [22] and values of the corresponding dimensionless parameters $\alpha^{2}$ and $\beta^{2}$. Data point presented in Fig. 1 of the manuscript.

Eq.(9) in the form:

$$
\begin{aligned}
& \ddot{\theta}(\tau)-\left(-\alpha^{2}-\beta^{2} \cos (\tau)\right) \theta(\tau)=0 \\
& \text { with } \alpha^{2}=\frac{g}{L} \frac{1}{\omega^{2}} \text { and } \beta^{2}=\frac{\Gamma}{\omega^{2}} \frac{1}{L} .
\end{aligned}
$$

For this system, an experimental example is presented in the work of Smith [22]. The length of the pendulum is $L=0.008 \mathrm{~m}$, the mass is $m=0.19 \times 10^{-3} \mathrm{~kg}$ and the gravitational acceleration is $g=9.81 \mathrm{~m} . \mathrm{s}^{-2}$. The dimensionless experimental parameters are calculated using the experimental values of Smith [22] and Eq.(11) and are shown in Table 3.

\section{A.4. Microelectromechanical systems: MEMS}

In this case parametric instabilities are used to amplify the movement of microelectromechanical membranes (MEMS). Jia et al. [13] used a Duffing oscillator to describe the motion of the membrane:

$$
\begin{array}{r}
\frac{d^{2} x(t)}{d t^{2}}+2 \zeta_{1} \omega_{m} \frac{d x(t)}{d t}+\zeta_{2}\left|\frac{d x(t)}{d t}\right| \frac{d x(t)}{d t}+ \\
b x^{3}+\left(\omega_{m}^{2}-\xi \Gamma \cos \omega t\right) x(t)=0
\end{array}
$$

where $x$ is the displacement of the membrane, $\omega_{m}$ is the natural frequency of the mode $m, \zeta_{1}$ is the viscous damping ratio, $\zeta_{2}$ is the nonlinear quadratic damping coefficient, $b$ is the mass normalised Duffing coefficient, $\xi$ is a standard coefficient relating the external excitation to the parametric excitation, $\omega$ is the frequency of excitation and $\Gamma$ is the acceleration of the excitation.

To compare this equation with our model we make the assumption that there is no damping and we neglect non linear terms, i.e.: we consider only small oscillations about the equilibrium position. Upon the change of variable $\tau=\omega t$, equation Eq.(12) can be expressed in the standard dimensionless form of the Mathieu equation of the manuscript:

$$
\begin{aligned}
& \ddot{x}(\tau)+\left(\alpha^{2}-\beta^{2} \cos (\tau)\right) x(\tau)=0 \\
& \text { with } \alpha^{2}=\frac{\omega_{m}^{2}}{\omega^{2}} \text { and } \beta^{2}=\Gamma \frac{\xi}{\omega^{2}} .
\end{aligned}
$$

The experimental parameters are the one used in the work of Jia et al. [13]. The natural frequency of the membrane is $f_{0}=980 \mathrm{~Hz}$ and the value of the standard coefficient is $\xi=1.7744 \times 10^{6}$. The dimensionless experimental parameters are calculated using the experimental values of Jia et al. [13] and Eq.(13) and are reported in Table 4. 


\begin{tabular}{|l|l|l|}
\hline Experimental parametric parameters & $\alpha^{2}$ & $\beta^{2}$ \\
\hline$f=1960 \mathrm{~Hz} ; \Gamma=0.4 \mathrm{~m} . \mathrm{s}^{-2}$ & 0.25 & 0.0047 \\
$f=980 \mathrm{~Hz} ; \Gamma=0.5 \mathrm{~m} . \mathrm{s}^{-2}$ & 1.0 & 0.023 \\
$f=650 \mathrm{~Hz} ; \Gamma=6 \mathrm{~m} \cdot \mathrm{s}^{-2}$ & 2.27 & 0.64 \\
$f=480 \mathrm{~Hz} ; \Gamma=8 \mathrm{~m} . \mathrm{s}^{-2}$ & 4.17 & 1.56 \\
\hline
\end{tabular}

\section{Table 4}

Experimental parameters used by Jia et al.[13] and values of the corresponding dimensionless modulation parameters $\alpha^{2}$ and $\beta^{2}$. Data points presented in Fig.1 of the manuscript.

\section{B. Triggering and sustaining a natural oscillation}

We present the experimental observation of a $12^{\text {th }}$ order parametric resonance using the "impulse train" scenario described in Section 4. The period of modulation is $T=6 T_{0}$ and current intensity $I=1.25 \mathrm{~A}$. To assure a minimal exchange of energy we set $T_{1}=0.99 T_{0}$ and $T_{2}=0.01 T_{0}$.

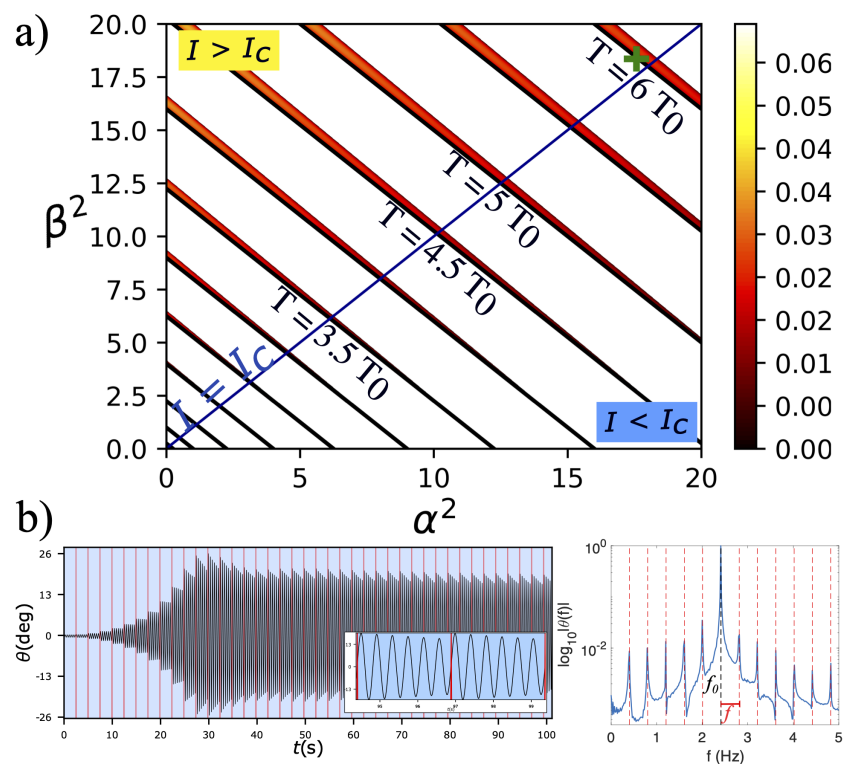

Figure 9: Triggering a "natural" limit cycle. a) Stability diagram of the Meissner equation Eq.(1) in the "impulse train" case when $T_{1}=0.99 T$ and $T_{2}=0.01 T$. Black lines show the limit $T_{1} \rightarrow T$ and $T_{2} \rightarrow 0$. The green cross represents the experimental modulation parameters associated with the response shown in b) Left: experimental response observed when the electromagnetic pendulum is OFF during $T_{1} \approx 2.46 \mathrm{~s}$ and $\mathrm{ON}$, with $I \approx 1.25 \mathrm{~A}\left(I>I_{c}\right)$ during $T_{2} \approx 0.025 \mathrm{~s}\left(\approx 0.01 T_{1}\right)$. Inset zooms on the $T$-periodic limit cycle. Red lines represent the very short time the magnets are ON. Right: Fast Fourier Transform of the permanent regime of the response. The black dashed-line represents the value of the natural frequency of the pendulum when the red ones represent the secondary harmonics $f_{0} \pm h f$, where $f=1 / T$ and $h$ is an integer.

Expressing the experimental parameters in the $\left(\alpha^{2}, \beta^{2}\right)$ dimensionless space, we report experimental data (green cross) in the stability diagram of Fig.9a). We find, as expected since the applied modulation period $T$ is $12^{\text {th }}$ time slower than half the natural period of the pendulum, that the limit cycle of Fig.9b) results from a parametric resonance of order $k=12$. Since $k$ is even, we expect to trigger a $T$-periodic limit cycle which is confirmed by the FFT of the experimental permanent regime in Fig.9b). Interestingly, the limit cycle is very close to a purely sinusoidal motion with a fundamental frequency $f_{0}$ since the amplitudes of the secondary harmonics weight no more than $3 \%$ of the fundamental one. Actually, as illustrated by the inset of Fig.9b) that displays the last two periods of the recorded experimental response, the triggered limit cycle is the free damped oscillation of the pendulum with natural period $T_{0}$ that is "reset" every $6 T_{0}$ thanks to the synchronized impulse of input energy represented by red vertical lines.

\section{References}

[1] John A Richards. Analysis of periodically time-varying systems. Springer Science \& Business Media, 2012.

[2] Juan R Sanmartin. O botafumeiro: Parametric pumping in the middle ages. American Journal of Physics, 52(10):937-945, 1984.

[3] Michael Faraday. On a peculiar class of acoustical figures; and on certain forms assumed by groups of particles upon vibrating elastic surfaces. Philosophical transactions of the Royal Society of London, 121:299-340, 1831.

[4] Thomas Brooke Benjamin and Fritz Joseph Ursell. The stability of the plane free surface of a liquid in vertical periodic motion. Proceedings of the Royal Society of London. Series A. Mathematical and Physical Sciences, 225(1163):505-515, 1954.

[5] Stéphane Douady. Experimental study of the faraday instability. Journal of Fluid Mechanics, 221:383-409, 1990.

[6] Raymond E Goldstein. Coffee stains, cell receptors, and time crystals: Lessons from the old literature. Physics Today, September 2018.

[7] VV Bolotin. The dynamic stability of elastic systems, 1964. ITIoldenDay, lnc, 1964.

[8] A Vafai, M Javidruzi, and HE Estekanchi. Parametric instability of edge cracked plates. Thin-walled structures, 40(1):29-44, 2002.

[9] Matthew Evans, Slawek Gras, Peter Fritschel, John Miller, Lisa Barsotti, Denis Martynov, Aidan Brooks, Dennis Coyne, Rich Abbott, Rana X Adhikari, et al. Observation of parametric instability in advanced ligo. Physical Review Letters, 114(16):161102, 2015.

[10] Alexey V Arefiev, Boris N Breizman, Marius Schollmeier, and Vladimir N Khudik. Parametric amplification of laser-driven electron acceleration in underdense plasma. Physical Review Letters, 108(14):145004, 2012.

[11] Kimberly L Turner, Scott A Miller, Peter G Hartwell, Noel C MacDonald, Steven H Strogatz, and Scott G Adams. Five parametric resonances in a microelectromechanical system. Nature, 396(6707):149$152,1998$.

[12] Alex Szorkovszky, Andrew C Doherty, Glen I Harris, and Warwick P Bowen. Mechanical squeezing via parametric amplification and weak measurement. Physical Review Letters, 107(21):213603, 2011.

[13] Yu Jia, Sijun Du, and Ashwin A Seshia. Twenty-eight orders of parametric resonance in a microelectromechanical device for multi-band vibration energy harvesting. Scientific reports, 6:30167, 2016.

[14] Frank Wilczek. Quantum time crystals. Physical Review Letters, 109(16):160401, 2012

[15] Dominic V Else, Bela Bauer, and Chetan Nayak. Floquet time crystals. Physical Review Letters, 117(9):090402, 2016.

[16] Toni L Heugel, Matthias Oscity, Alexander Eichler, Oded Zilberberg, and R Chitra. Classical many-body time crystals. Physical Review Letters, 123(12):124301, 2019

[17] Barend Bentvelsen and Arnaud Lazarus. Modal and stability analysis of structures in periodic elastic states: application to the ziegler column. Nonlinear Dynamics, 91(2):1349-1370, 2018.

[18] Arnaud Lazarus. Discrete dynamical stabilization of a naturally di- 


\section{Extreme parametric instabilities}

verging mass in a harmonically time-varying potential. Physica D: Nonlinear Phenomena, 386:1-7, 2019.

[19] Benjamin Apffel, Filip Novkoski, Antonin Eddi, and Emmanuel Fort. Floating under a levitating liquid. Nature, 585:48-52, 2020.

[20] Andrew Stephenson. Xx. on induced stability. The London, Edinburgh, and Dublin Philosophical Magazine and Journal of Science, 15(86):233-236, 1908.

[21] DJ Acheson. Upside-down pendulums. Nature, 366:215-216, 1993.

[22] HJT Smith and James A Blackburn. Experimental study of an inverted pendulum. American Journal of Physics, 60(10):909-911, 1992.

[23] Suzie Protiere. Gouttes rebondissantes: une association ondeparticule à échelle macroscopique. $\mathrm{PhD}$ thesis, Paris Diderot, 2007.

[24] Balth van der Pol and MJO Strutt. Ii. on the stability of the solutions of mathieu's equation. The London, Edinburgh, and Dublin Philosophical Magazine and Journal of Science, 5(27):18-38, 1928.

[25] Chikara Sato. Correction of stability curves in hill-meissner's equation. Mathematics of Computation, 20(93):98-106, 1966.

[26] Protière S. Grandi, A.A. and Lazarus A. Movie 1 showing the experimental response of the electromagnetic pendulum periodically turned in an oscillating state $\omega(0)=\sqrt{g / l}=15.24 \mathrm{rad} / \mathrm{s}$ during $T_{1}=0.31 \mathrm{~s}$ and $\omega(I)=6.8 \mathrm{rad} / \mathrm{s}$ during $T_{2}=0.31 \mathrm{~s}$. Movie 2 showing the experimental response of the electromagnetic pendulum periodically turned in an oscillating state $\omega(0)=\sqrt{g / l}=15.24 \mathrm{rad} / \mathrm{s}$ during $T_{1}=5.08 \mathrm{~s}$ and $\omega(I)=6.5 \mathrm{rad} / \mathrm{s}$ during $T_{2}=5.08 \mathrm{~s}$. Movie 3 showing the experimental response of the electromagnetic pendulum periodically turned in an oscillating state $\omega(0)=\sqrt{g / l}=15.24 \mathrm{rad} / \mathrm{s}$ during $T_{1}=1.24 \mathrm{~s}$ and a diverging state $\omega^{2}(I)=-1.1(\mathrm{rad} / \mathrm{s})^{2}$ during $T_{2}=0.025 \mathrm{~s}$.

[27] Dian Yang, Bobak Mosadegh, Alar Ainla, Benjamin Lee, Fatemeh Khashai, Zhigang Suo, Katia Bertoldi, and George M Whitesides. Buckling of elastomeric beams enables actuation of soft machines. Advanced Materials, 27(41):6323-6327, 2015.

[28] Douglas P Holmes and Alfred J Crosby. Snapping surfaces. Advanced Materials, 19(21):3589-3593, 2007. 